\title{
Fetal Wound Healing
}

Magda M.W.Ulrich

\section{Contents}

1.1 Background - 4

1.2 Inflammation - 4

1.3 Extracellular Matrix - 5

1.4 Angiogenesis - 5

$1.5 \quad$ Keratinocytes -6

1.6 Fibroblasts -7

1.7 Mechanical Forces - 7

1.8 Remodeling - 8

1.9 Skin Appendix Formation - 8

1.10 Conclusions -8

References - 9 


\subsection{Background}

Wound healing is a complex and tightly regulated process and can be divided in three different overlapping phases: the inflammatory phase, the proliferation phase, and the remodeling phase. The first phase begins directly after wounding and is characterized by hemostasis and inflammation. Platelets play an important role in the formation of the blood clot to stop the bleeding, and through the secretion of chemokines, they recruit different inflammatory cells such as neutrophils and monocytes to the wound. The inflammatory cells clean the wound by phagocytosis of invading microorganisms and damaged tissue. The inflammatory cells also secrete chemotactic signals which attract fibroblasts and endothelial cells to the wound which, together with the inflammatory cells, form the granulation tissue. This is the proliferation phase during which the destroyed tissue is replaced. During this phase, the keratinocytes migrate over the granulation tissue to form a new epidermis to close the skin defect.

The last phase in wound healing is the remodeling phase which is characterized by a decrease in cellularity and vascularity by apoptosis and an increase of synthesis and deposition of the components of the extracellular matrix $(\mathrm{ECM})$. In adults, this process results is scar formation, whereas in fetal skin, complete regeneration of the skin, including the formation of skin appendices such as hairs and sebaceous and sweat glands, takes place.

Since the 1950, studies on scarless healing in fetal skin in animals have been published, and in the late 1970s of the last century, the first publications appeared describing scarless healing of fetal skin wounds in humans. Since then, several experimental studies on this subject have been published. From experimental animal and human studies it is known that scarless healing only occurs during the first period of gestation. Human fetuses lose the ability of skin regeneration around 22 weeks of gestation.

Animal experiments in which adult, late gestational, and early gestational fetal skin were transplanted on early gestational fetuses showed clear differences in wound healing outcome. Incision wounds created in these transplants showed scar formation in adult and late gestational fetal skin, whereas the wound created in the fetal skin resulted in complete regeneration [1]. These experiments show not only that this phenomenon is an intrinsic property of the fetal skin itself but also that scarless healing is not triggered by the intrauterine environment. Interestingly, the wounds at the fetal-adult interface also healed without scar formation implicating that direct contact of a wound with fetal skin is sufficient to induce regeneration of the tissue [2].

Several mechanisms have been suggested to be responsible for the scarless healing in fetal skin, e.g., a diminished inflammatory reaction, differences in composition and architecture of the extracellular matrix, and mechanical load in fetal skin. Furthermore, fetal skin differs from adult skin in many factors commonly associated with scar formation such as proteolytic activity and TGF- $\beta$ secretion and sensitivity. This chapter will discuss the different mechanisms during the three phases of wound healing proposed in the literature to be involved in scarless healing.

\subsection{Inflammation}

Several studies have shown that the inflammatory response to injury is strongly reduced in fetal skin wounds, and induction of the local inflammation causes scar formation in early fetal skin.

The inflammatory reaction involves recruitment of inflammatory cells which produce inflammatory mediators such as growth factors and cytokines. The growth factors and cytokines subsequently recruit more inflammatory cells.

Inflammation in adult wound healing starts off with a pro-inflammatory cytokine and immune cell response and the activation of the acute phase response. During this period, invading microorganisms and injured tissue are cleared from the wound bed. Subsequently, the inflammatory reaction is skewed to an anti-inflammatory profile which initiates the healing response. Although the exact role of inflammatory cells in scar formation is not entirely elucidated, it is evident that they play an important role in the derailed wound healing process leading to scar formation. It was shown that in severely scarred wounds such as burn wounds, the acute (pro-) inflammatory status is continued for a very long time.

Cells involved in the pro-inflammatory reaction are neutrophils and the M1 subtype macrophages, while macrophages of the M2 subtypes and regulatory $\mathrm{T}$ cells are anti-inflammatory, pro-healing cells.

Platelets are the first cells involved in the induction of the inflammatory reaction. The platelets are activated and aggregate during the coagulation phase forming the hemostatic plug. The activated platelets release cytokines such as platelet-derived growth factor (PDGF), interleukins (IL) 1 and 6, and transforming growth factor beta (TGF- $\beta$ ) into the wound environment. It was shown that fetal platelets aggregate poorly when exposed to higher levels of hyaluronic acid, a component of the extracellular matrix, which is more abundantly expressed in fetal skin than in adult skin, and subsequently release lower levels of platelet-derived cytokines.

The lower platelet-derived cytokines are most likely responsible for the reduced inflammatory cell infiltration, such as neutrophils, macrophages, and lymphocytes, seen in fetal wounds at early gestational age [3]. 
Not only recruitment of inflammatory cells from the circulation to the fetal wound is reduced, but also the number of resident macrophages, mast cells, Langerhans cells, dendritic cells and T cells in uninjured fetal skin is reduced. Moreover, the immune cells present in the fetal skin have the anti-inflammatory, pro-healing phenotype as fetal skin contains higher numbers of regulatory $\mathrm{T}$ cells and macrophages of the M2 phenotype. In addition, chemoattractant cytokines such as CCL17, CCL21, and CCL27 involved in recruitment of T cells and dendritic cells are reduced in fetal skin [4].

Although the inflammatory growth factor TGF- $\beta$ plays an important role in scar formation, it is also involved in the resolution of the inflammatory reaction. In fetal skin, high levels of all the components of the TGF- $\beta$ pathway are present, and moreover, the pathway seems to be highly activated. This highly activated TGF- $\beta$ pathway might also result in fast suppression of the pro-inflammatory reaction in fetal wounds [4].

These findings indicate that indeed the (pro-)inflammatory immune reactions is reduced in fetal wound healing. However, the inflammatory component cannot be the key link to scarless healing since wounding of transplanted adult sheep skin to a sheep fetus resulted in scar formation despite the diminished inflammatory state of the fetus itself and the intrauterine environment.

\subsection{Extracellular Matrix}

The extracellular matrix of fetal skin is distinctly different than adult skin matrix. Early gestational fetal skin contains higher amounts of collagen type III, fibronectin EIIIA, and hyaluronic acid (HA) and is deficient in elastin. Similar expression profiles for ECM components were demonstrated for the (adult) oral mucosa, a tissue also known for reduced scar formation.

Although collagen type I is the major component in both adult and fetal skin, in fetal skin, collagen type III/ collagen type I ratio is higher compared to adult skin. The higher levels of collagen type III have effects on the ECM organization and collagen fiber diameter. Downregulation of collagen type III expression results in increased scar formation and differentiation of fibroblasts into myofibroblasts, the main cell type involved in scar formation [5].

The properties of the ECM network do not depend only on the presence and percentage of the different collagen subtypes and other ECM components but also on cross-linking of the ECM components. Increased crosslinking results in ECM accumulation and increased rigidity. In fetal skin, most of the collagen fibers are not cross-linked [5]. One of the enzymes involved in collagen and elastin cross-linking is lysyl oxidase (LOX). Early gestational fetal wounds show reduced LOX expression in comparison to late gestational wounds.
Other important components of the ECM which are differentially expressed between fetal and adult skin are glycosaminoglycans (GAGs) and proteoglycans. The GAGs like hyaluronic acid (HA) contain long polysaccharide chains which can absorb large amounts of water and are important for hydration of the skin and determine the viscoelasticity of the skin. HA is thought to play an important role in regulation of the inflammatory reaction during wound healing. However, its effect is dependent on the length of the polysaccharides. High-molecular-weight (HMW) HA suppresses the inflammatory reaction, while low-molecular-weight (LMW) HA fragments are immunostimulatory and induce the inflammatory reaction [6].

Expression of HA in fetal skin is high and during wound healing the expression is induced in adults. The positive effect in fetal wound healing might be related to the balance between HMW and LMW HA, which may be caused by differential expression of enzymes synthesizing HA (hyaluronan synthases) and enzymes degrading HA (hyaluronidases) between adult and fetal tissue. It was shown that addition of hyaluronidases to fetal wounds can induce fibroplasia, collagen deposition, and neovascularization, processes involved in scar formation.

Besides the abovementioned component, elastin is also an important component of the ECM. This protein is responsible for connective tissue pliability in the adult dermis; however, it is absent in early gestational skin, and its role in scarless wound healing is not clear [7] (• Fig. 1.1).

\subsection{Angiogenesis}

Several studies have shown that reduced angiogenesis during adult wound healing reduces scar formation. However, indisputable evidence that this also plays a role during fetal wound healing has not been provided although several studies suggested lack of strong neovascularization and reduced proangiogenic factors in fetal wounds.

Angiogenesis is much more prominent in adult wound healing compared to fetal wound healing. Early in adult wound healing, a dense capillary bed is created. However, this capillary tangle lacks the assembly of a functional network which is able to provide nutrition and oxygen to the wound bed [8].

It was shown that HA degradation products induce angiogenesis; therefore, the reduced synthesis of hyaluronidases not only has a positive effect on suppression of the inflammatory reaction but also reduces the exaggerated neovascularization seen in adult wounds.

However, this could also be attributed to reduced levels of angiogenic growth factors such as basic fibroblast growth factor and PDGF. Addition of angiogenic factors such as hyaluronidase and PDGF to fetal wounds was shown to induce angiogenesis and lead to scar formation. 


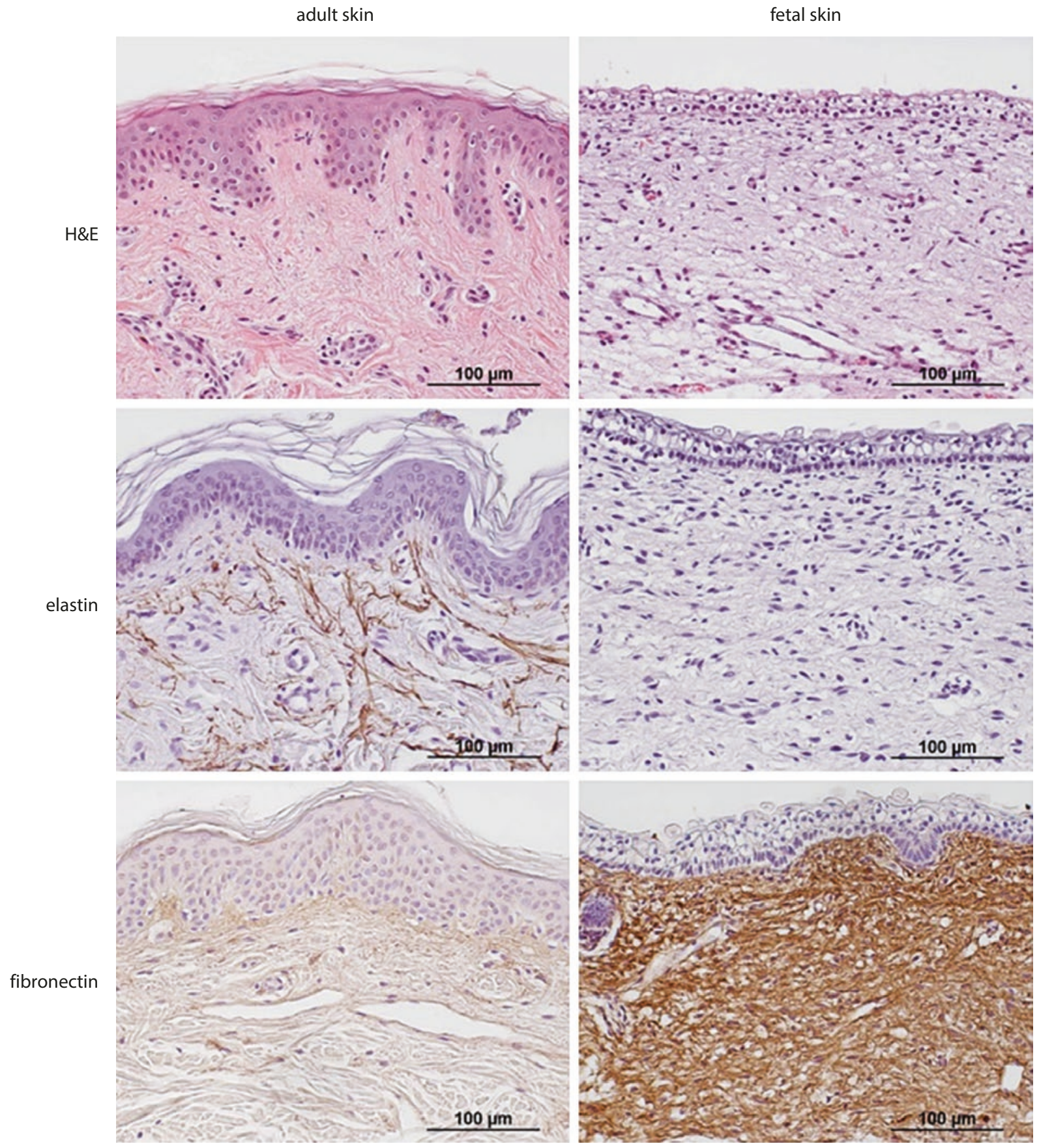

- Fig. 1.1 Expression of different ECM components in adult and fetal skin. The specific ECM components are stained in brown

\subsection{Keratinocytes}

One of the factors thought to play a role in scar formation is delayed wound closure by reepithelialization. Wound closure in fetal wounds is much faster in comparison to adult wounds.

Fetal keratinocytes not only have a higher proliferation rate; also the mechanism of reepithelialization was found to be distinctly different between adult and fetal wounds. In adult wound healing reepithelialization, the basal keratinocytes at the wound edge undergo morphological changes. They form actin containing cytoplasmic elongations called lamellipodia through which they crawl over the extracellular matrix of the wound bed. To be able to migrate, the cells lose their ECM binding structures (hemidesmosomes) and the cell-cell binding sites (desmosomes). Fetal keratinocytes do not form lamellipodia but form an actin myosin II containing 
cables through the front keratinocytes at the wound edge [9]. This cable runs from cell to cell most likely through the adherens junctions, another form of cell-cell junction similar to the desmosome. The cable is formed within hours after wounding and by contraction pulls the keratinocytes together to close the wound.

Integrins may also play a role in faster reepithelialization in fetal wound healing. Integrins are heterodimeric transmembrane receptors which can bind to specific ECM components. Different combinations of the subunit determine the specificity for the different ECM components, and they determine cellular processes like cell proliferation and migration. In the fetus, it was shown that specific integrins were rapidly upregulated upon wounding and that this occurred much faster than in adult keratinocytes.

It is evident that there is an interaction between keratinocytes and fibroblasts in the dermis. In vitro experiments have shown that fetal keratinocytes suppress proliferation, myofibroblast differentiation, and ECM production, in fibroblasts derived from hypertrophic scar, whereas fetal keratinocytes promote proliferation and migration in fetal and adult healthy skin-derived fibroblasts.

\subsection{Fibroblasts}

Fibroblasts are the main cell type of the dermis; they produce the ECM and therefore play an important role in wound healing and scar formation. During wound healing, this cell type differentiates into myofibroblasts. These latter cells produce high levels of ECM components and contain the contractile protein alpha smooth muscle actin $(\alpha \mathrm{SMA})$ which is involved in tissue contraction seen in scar formation. The transition to a myofibroblast population in the wound healing environment and especially the persistence of this cell population are seen as the most important contributor to scar formation. Transforming growth factor beta (TGF- $\beta$ ) plays an important role in this process. However, TGF- $\beta$ also plays an important role during embryonic and fetal development and thus is abundantly present in the fetus. TGF- $\beta$ is stored as an inactive compound in the ECM. In order to execute its role in the many processes this growth factor is involved in, it has to be activated which ultimately leads to the induction of various genes including genes involved in fibrosis and the process of scar formation. It was shown that all of the components are abundantly present in the fetal skin. In addition, the TGF- $\beta$ pathway was also shown to be highly activated in healthy fetal skin in comparison to adult skin, but this does not result in fibrotic skin or scar formation. It was suggested that differential expression in TGF- $\beta$ isoforms plays a crucial role in this. TGF- $\beta 1$ and TGF- $\beta 2$ are thought to be profibrotic, whereas TGF- $\beta 3$ has antifibrotic properties. However, this segmentation is not completely indisputable. The balance between the profibrotic and antifibrotic TGF- $\beta$ isoforms in fetal skin is shifted in comparison to adult skin: $99.5 \%: 0.5 \%$ in adult to $97.4 \%: 2.6 \%$ in fetal skin. However, the absolute concentrations of all TGF- $\beta$ isoforms are much higher in fetal skin in comparison to adult skin.

Several differences were observed between fetal skin fibroblasts and adult dermal fibroblasts: The fetal cells migrated much faster than adult fibroblasts and exhibited a "hyperactive morphology." They produced higher amounts of extracellular matrix components. Furthermore, fetal cells produced a larger contraction effect when cultured in fibroblast-populated collagen lattices compared to adult fibroblasts.

As in fetal keratinocytes, it was shown that fetal fibroblasts express different integrins which results in higher proliferation and migration rates. Inhibition of one of the specific fetal integrins resulted in a more adultlike fibroblast phenotype.

Stimulation of fetal fibroblasts in vitro also induced myofibroblast differentiation and shows higher contraction of fibroblast-seeded collagen matrices [4].

Studies in which fetal skin cells have been applied to burn wounds show improved wound healing. Since it was shown that the fetal cells were not incorporated into the newly formed skin, it is thought that the cells secrete growth factors or in any other form interact with the host organism to accelerate wound healing.

\subsection{Mechanical Forces}

Mechanical forces, extracellular as well as intracellular, play an important role in skin homeostasis as it directs cell function and activity through a process called mechanotransduction. In this process, the composition, viscoelasticity, and stiffness of the ECM play an important role. Adult skin contains thick collagen bundles mainly consisting of type I fibrils, whereas fetal skin contains thin collagen fibers which are high in collagen type III fibrils and show reduced resting stress.

Through adhesion molecules on the surface of the skin, the integrins, the ECM is connected to the cytoskeleton of the cell. The link between the ECM and the cytoskeleton is a dynamic complex, called focal adhesions (FA), which are constantly assembled and disassembled. The FA are able to direct not only cell function such as gene expression but also cellular adhesion and cell migration. Fibroblasts exposed to mechanical stress form supermature FA displaying a specific composition of FA proteins such as paxilin (Pax). Fetal fibroblasts 
were shown to have reduced FA protein expression among which Pax and different integrin subunits [4].

Also from clinical data, there is evidence that mechanical stress plays an important role in scar formation. Collagen fibers in the ECM of the skin are aligned in a specific pattern depending on the location of the body. This pattern forms the so-called Langer lines and determines the direction of the tension in the skin. From surgery, it is known that incisions made parallel to these lines heal with less scar formation than incisions perpendicular to the Langer lines, suggesting that mechanical load to the wound influences scar formation [10]. In fetal skin, the ECM is organized in a loose reticular network of collagen, while in adult skin, there are thicker more compact collagen fibers. In fetal wound healing, the same reticular ECM network is formed indistinguishable from the unaffected surrounding, whereas in adult wound healing, the collagen fibers are arranged in dense parallel bundles [11].

\subsection{Remodeling}

The last phase in wound healing is the remodeling phase. During this phase, cellularity decreases by apoptosis, and the degradation of the excessive extracellular matrix takes place. In adult wound healing, the predominant type III collagen is replaced by type I collagen. However, in fetal recovered skin, type III collagen remains the main subtype.

The deposition of extracellular matrix is an interplay between synthesis and degradation of the components of the ECM. Matrix metalloproteinases (MMPs) and tissue inhibitors of metalloproteinases (TIMPs) play an important role in this process. The MMP family consists of more than 20 members which are able to degrade most of the components of the ECM with different specific activities toward the different components. The process of ECM degradation is controlled by specific tissue inhibitors of metalloproteinases (TIMPs). Currently, four TIMPs have been identified which possess varying affinity to specific MMPs. An imbalance between MMPs and TIMPs affects the deposition of ECM. In scarring, reduced collagen degradation as a result of reduced MMP expression and increased TIMP expression causes accumulation of ECM components. Early gestational fetal skin and middle gestational fetal skin express lower MMP and TIMP levels than late gestational skin and adult skin. During fetal wound healing, several MMPs were increased in early gestational fetal wounds, whereas later during gestation, when wounds heal with scar formation, MMP levels were reduced, and TIMP levels were increased [12]. This later finding may explain the accumulation of ECM seen in scar formation.

\subsection{Skin Appendix Formation}

Early gestational wound healing results not only in restoration of the dermis and epidermis but also in the regeneration of skin appendages such as hair follicles and sebaceous and sweat glands.

Hair follicles contain several stem cell niches and play an important role in adult wound healing. In partial-thickness wounds in which part of the hair follicle is still intact, healing can occur without scar formation.

Hair follicle development during embryogenesis starts, depending on the anatomical location, between weeks 9 and 15 . One could envision that early gestational skin contains stem cells and still has the mechanisms available to induce skin appendage development and create the stem niches but that these stem cells can also regenerate the skin. It was shown that allogeneic cells derived from fetal skin could enhance burn wound healing and reduce scar formation.

\subsection{Conclusions}

The fact that adult skin in an intrauterine environment still heal with scar formation despite the low inflammatory milieu, the presence of fetal growth factors, and even fetal cells able to migrate into the adult tissue shows that the processes leading to scarless healing or scar formation cannot be attributed to single individual mechanisms, cells, or other factors but that it is a result of complex of interconnected processes.

\footnotetext{
Take-Home Messages

- Early to midgestational skin wounds result in skin regeneration instead of scar formation.

- The exact mechanism behind scarless healing is still unknown.

- Several mechanisms have been indicated: reduced inflammation, altered cytokine profile, and different ECM composition.

- The process of scarless healing is a result of a complex of interconnected processes.

- Several characteristics of fetal skin are shared with oral mucosa which is also known for its reduced scar formation.
} 


\section{References}

1. West DC, Shaw DM, Lorenz P, Adzick NS, Longaker MT. Fibrotic healing of adult and late gestation fetal wounds correlates with increased hyaluronidase activity and removal of hyaluronan. Int J Biochem Cell Biol. 1997;29(1):201-10. Epub 1997/01/01.

2. Sullivan KM, Meuli M, MacGillivray TE, Adzick NS. An adultfetal skin interface heals without scar formation in sheep. Surgery. 1995;118(1):82-6. Epub 1995/07/01.

3. Wilgus TA. Regenerative healing in fetal skin: a review of the literature. Ostomy Wound Manage. 2007;53(6):16-31; quiz 2-3. Epub 2007/06/26.

4. Walraven M. Cellular and molecular mechanisms involved in scarless wound healing in the fetal skin. Amsterdam: VU university medical center; 2016. https://research.vu.nl/ws/portalfiles/ portal/42155796/complete+dissertation.pdf.

5. Moore AL, Marshall CD, Barnes LA, Murphy MP, Ransom RC, Longaker MT. Scarless wound healing: Transitioning from fetal research to regenerative healing. Wiley Interdiscip Rev Dev Biol. 2018;7(2):e309. Epub 2018/01/10.

6. Ghatak S, Maytin EV, Mack JA, Hascall VC, Atanelishvili I, Moreno Rodriguez R, et al. Roles of proteoglycans and glycosaminoglycans in wound healing and fibrosis. Int $\mathrm{J}$ Cell Biol. 2015;2015:834893. Epub 2015/10/09.

7. Li M, Zhao Y, Hao H, Han W, Fu X. Theoretical and practical aspects of using fetal fibroblasts for skin regeneration. Ageing Res Rev. 2017;36:32-41. Epub 2017/02/28.

8. DiPietro LA. Angiogenesis and wound repair: when enough is enough. J Leukoc Biol. 2016;100(5):979-84. Epub 2016/11/02.

9. Redd MJ, Cooper L, Wood W, Stramer B, Martin P. Wound healing and inflammation: embryos reveal the way to perfect repair. Philos Trans R Lond B Biol Sci. 2004;359(1445):777-84.

10. Barnes LA, Marshall CD, Leavitt T, Hu MS, Moore AL, Gonzalez JG, et al. Mechanical forces in cutaneous wound healing: emerging therapies to minimize scar formation. Adv Wound Care. 2018;7(2):47-56. Epub 2018/02/03.

11. Lo DD, Zimmermann AS, Nauta A, Longaker MT, Lorenz HP. Scarless fetal skin wound healing update. Birth Defects Res C Embryo Today. 2012;96(3):237-47. Epub 2012/10/31.

12. Chen W, Fu X, Ge S, Sun T, Sheng Z. Differential expression of matrix metalloproteinases and tissue-derived inhibitors of metalloproteinase in fetal and adult skins. Int $\mathbf{J}$ Biochem Cell Biol. 2007;39(5):997-1005. Epub 2007/04/06.

Open Access This chapter is licensed under the terms of the Creative Commons Attribution 4.0 International License (http://creativecommons. org/licenses/by/4.0/), which permits use, sharing, adaptation, distribution and reproduction in any medium or format, as long as you give appropriate credit to the original author(s) and the source, provide a link to the Creative Commons license and indicate if changes were made.

The images or other third party material in this chapter are included in the chapter's Creative Commons license, unless indicated otherwise in a credit line to the material. If material is not included in the chapter's Creative Commons license and your intended use is not permitted by statutory regulation or exceeds the permitted use, you will need to obtain permission directly from the copyright holder. 